\title{
APPLICATION MAXILLOFACIAL MICROSURGERY AT HANOI NATIONAL HOSPITAL OF ODONTO-STOMATOLOGY
}

\author{
Nguyen Hong Nhung*, Nguyen Tan Van*, Le Duc Thanh*, Nguyen Tai Son*
}

\section{ABSTRACT}

Background: Microsurgery is a highly complex technique that requires specialized equipment and steady surgical techniques. This is a retrospective review of the development progress of microsurgical techniques at Hanoi National Hospital of Odonto-Stomatology (NHOS) during the past 12 years. Methods: Retrospective study assessing the training and developing process of microsurgical techniques at NHOS in 12 years. Results: NHOS has performed 665 microsurgical flaps reconstructions after surgical removal of large tumors in maxillomandibular area. 441 fibular free flaps were harvested for mandibular reconstructions after resections of ameloblastoma and osteofibroma. 4 fibular flaps were used for maxillary reconstructions. 119 osteocutaneous fibular free flaps were harvested for mandibular reconstructions in oral cancer surgeries. 83 lateral arm flaps and 13 anterolateral thigh flaps reconstructions were performed following cancer ablations. 5 patients underwent reconstruction after cancer resection with 2 fibular free flaps and anterolateral thigh flaps. Success rate is $654 / 665$ (98.35\%). Conclusion: The implementation of microsurgery technique at NHOS has been carried out for only 12 years but rapid and significant progress has been made. Free flap reconstruction is a flexible reconstructive option to restore essential functions and aesthetics of the facial area and to improve the patient's quality of life.

\footnotetext{
* Hanoi National Hospital of OdontoStomatology

Responsible person: Nguyen Hong Nhung

Email: dr.rosy245@gmail.com

Date of receipt: 01/6/2021

Date of scientific judgment: 15/7/2021

Reviewed date: 19/8/2021
}

\section{BACKGROUND}

Hanoi National Hospital of OdontoStomatology (NHOS) is a large facility specializing in Odonto-Stomatology and Oral surgery. Reconstructive intervention after surgical removal of diseased area is very important. The resection of large benign tumors or malignant tumors always leaves big defects that cannot be repaired with conventional reconstructive techniques.

The advent of microsurgery has encouraged surgeons to use free flaps to overcome the challenges faced by reconstructive surgery using conventional techniques. Microsurgical techniques were originally developed since the 1970s of the last century. In the 1980s, Dr. Nguyen Huy Phan developed microsurgical techniques in Vietnam. The first microsurgeries were performed at 108 Military Central Hospital. The NHOS took initiatives to perform the first microsurgeries in 2008.

\section{MATERIALS AND METHODS}

In this review, we report the progress of surgical approach, training and development of microsurgery at NHOS in the period from 2008 to 2020. We access the number of performed microsurgeries, types of free flaps, pathological conditions that require microsurgery, complications and surgical outcomes, as well as the success rate after surgery. 


\section{RESULTS AND DISCUSSION}

In 1984, NHOS set up an operating room for experimental microsurgery. As part of technology transfer program, the hospital cooperated with Mayo Clinic, USA, in 2005 and Faculty of Maxillofacial and Reconstructive Surgery of 108 Military Central Hospital in 2006. The surgeons of
NHOS perform first mandibular reconstructions with fibular flaps in 2008. In 2016, the hospital established a technology transfer cooperation with EDA Hospital, Taiwan. NHOS has performed 665 microsurgeries in total, of which 654/665 cases have successful outcomes.

Table 1: Total number of microsurgeries performed in period from 2008 to 2020

\begin{tabular}{|c|c|c|c|}
\hline Free Flaps & $\begin{array}{c}\text { Number of } \\
\text { cases }\end{array}$ & $\begin{array}{c}\text { Successful } \\
\text { Outcome }\end{array}$ & Flap loss \\
\hline Fibular flap & 564 & 556 & 8 \\
\hline Lateral arm flap & 83 & 80 & 3 \\
\hline Anterolateral thigh flap & 13 & 13 & 0 \\
\hline $\begin{array}{l}\text { Fibular free flap } \\
+ \text { Anterolateral thigh flap }\end{array}$ & 5 & 5 & 0 \\
\hline Total & 665 & $654(98,34 \%)$ & $11(1,65 \%)$ \\
\hline
\end{tabular}

Venous thrombosis is the most common complication after reconstructive surgery. Flap necrosis was observed in 8 cases due to complications of venous thrombosis on the 2nd day after surgery. Other 2 cases of flap failure were reported on 3rd day after surgery due to complications of arterial thrombosis. In order to reduce thromboembolic complications, patients received $325 \mathrm{mg}$ of Aspirin on the first day after surgery. One patient with diabetes experienced flap failure and non-healing wound due to arterial thrombosis complications.

Hanoi National Hospital of OdontoStomatology (NHOS) is a large facility specializing in Odonto-Stomatology and Oral surgery. Patients with large benign tumors (ameloblastoma, osteofibroma ...) frequently visit the hospital. The number of cancer patients coming for examination and treatment is also high. Thus, it is necessary to have a good surgical plan to treat the diseases while maintaining patient's quality of life. The most commonly encountered benign tumors in the jaw area are ameloblastomas. The tumors can be located either in the maxilla or mandible, more frequently in the mandible. These lesions are invasive with high risk of recurrence if not completely resected. The most common benign neoplasia that originated from bone are osteofibromas. These slow-growing tumors can cause facial deformities when they grow too large and they are likely to regrow if not removed completely. Effective treatment of large benign tumors often requires maxillomandibular osteotomy that leave large bone defects to be reconstructed. We have performed reconstructions of mandible using 441 fibular flaps and reconstructions of maxilla using 4 fibular flaps after resection of osteofibromas and ameloblastomas in these areas. 


\section{VIETNAM MEDICAL JOURNAL №1\&2/2021}
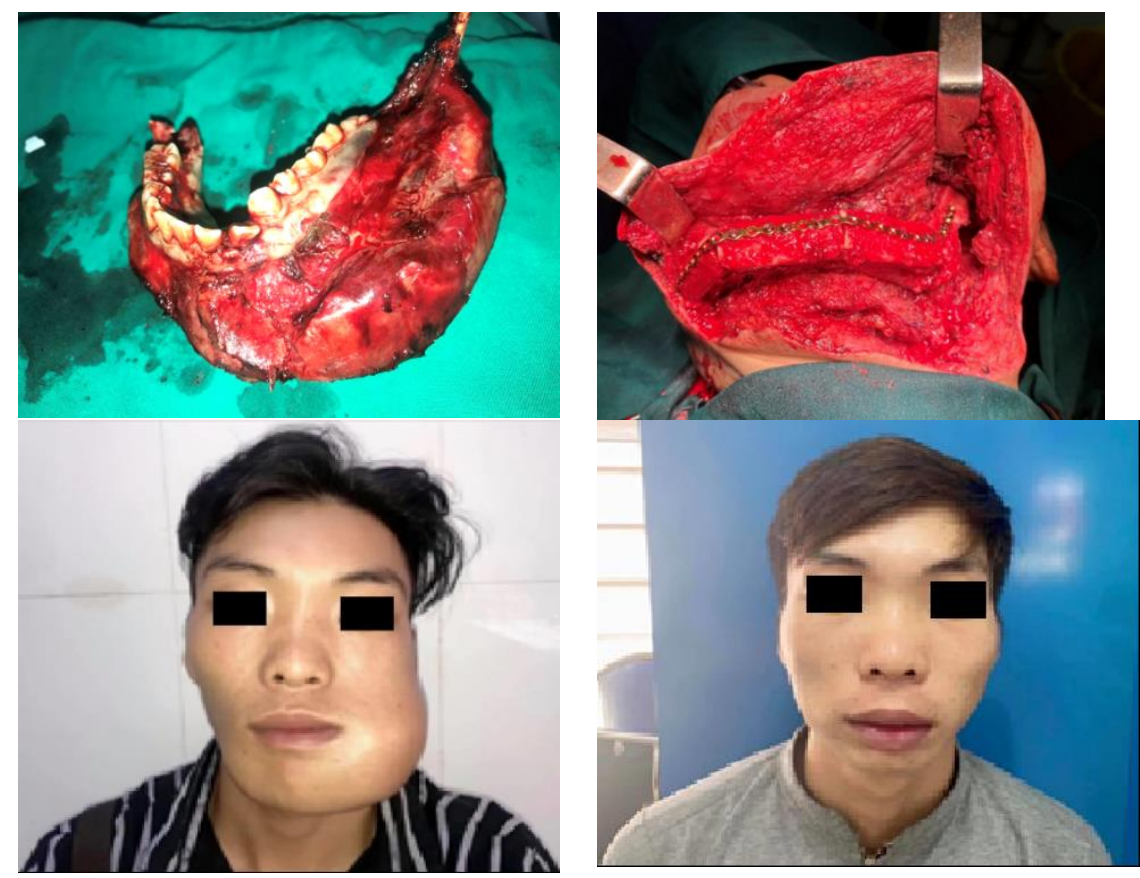

Figure 1. Mandibular recontruction with fibular flap after tumor resection.

Oral cancer is the 6th most common malignancy worldwide. In Vietnam, afflicted patients often visit hospitals for diagnostic and treatment with advanced-stage and large tumors. Oral cancer does not respond well to radiation or chemotherapy, so extensive tumor resection and cervical lymphadenectomy are the best treatment options. Wide surgical excisions of tumor often leave major maxillomandibular defects such as mandibular-oral floor defect, oral floor defect, partial or total tongue defect, tongue-oral floor defect, cheek mucosa defect, orofacial fistula. The reconstruction of these defects requires careful selection of suitable free flaps. We have harvested 119 osteocutaneous fibular free flaps to reconstruct the mandibular and to cover the oral floor; 83 lateral arm flaps for reconstructions of tongue, oral floor, buccal mucosa; 13 anterolateral thigh flaps to reconstruct major soft-tissue defects and to cover orofacial fistulas. The combination of both fibular flap and anterolateral thigh flaps is used in 5 cases to reconstruct mandibleoral floor and to cover the area after tumor resection.

Table 2. Classification of defects after tumor resection.

\begin{tabular}{|c|c|c|}
\hline Type of defect & Number of cases & Harvested free flaps \\
\hline Maxillary & 4 & Fibular flap \\
\hline Mandibular & 441 & Fibular flap \\
\hline Mandiblular + Mucosal & 119 & Skin flap - Fibular flap \\
\hline Soft tissue & 96 & Lateral arm flap, anterolateral thigh flap \\
\hline Skin - mandibular - Mucosal & 5 & \\
\hline Total & 665 & \\
\hline
\end{tabular}



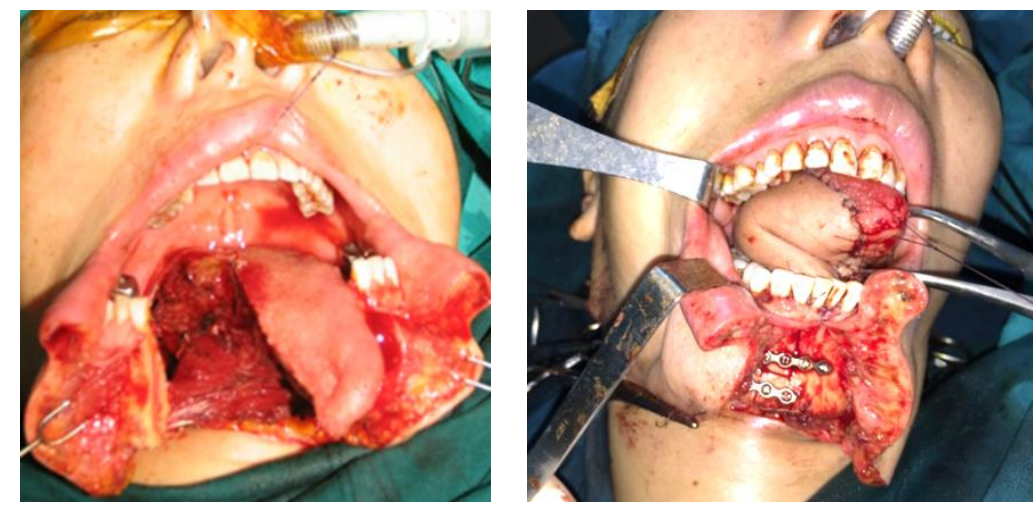

Figure 2. Tongue reconstruction with lateral arm flap.

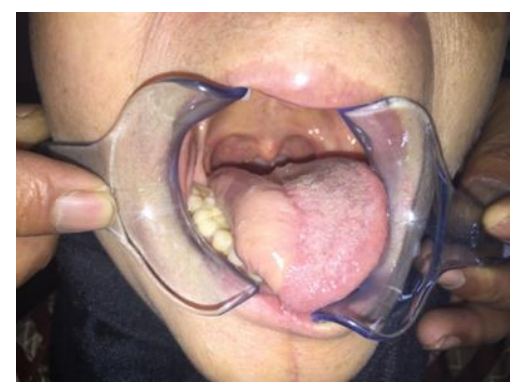

Figure 3. 6 months after tongue reconstruction.
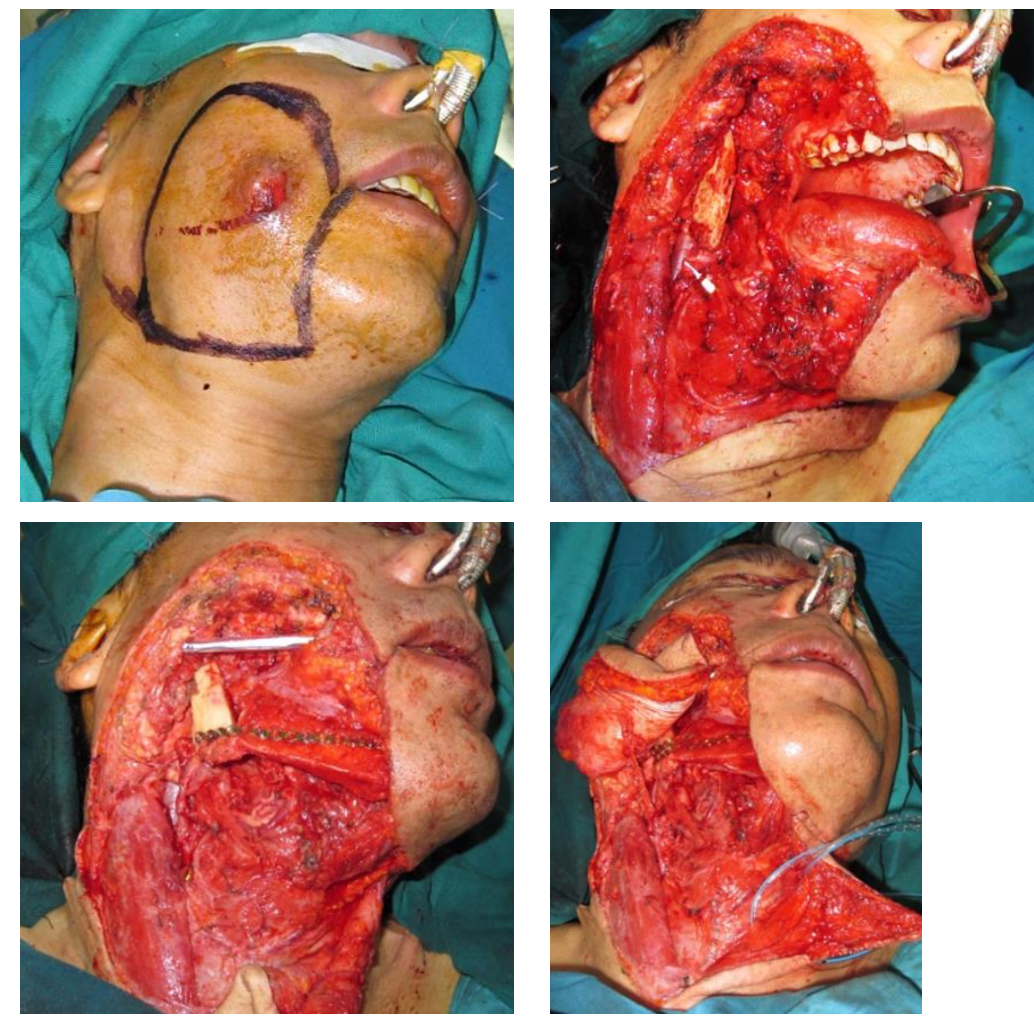


\section{VIETNAM MEDICAL JOURNAL No$^{\circ} \& 2 / 2021$}
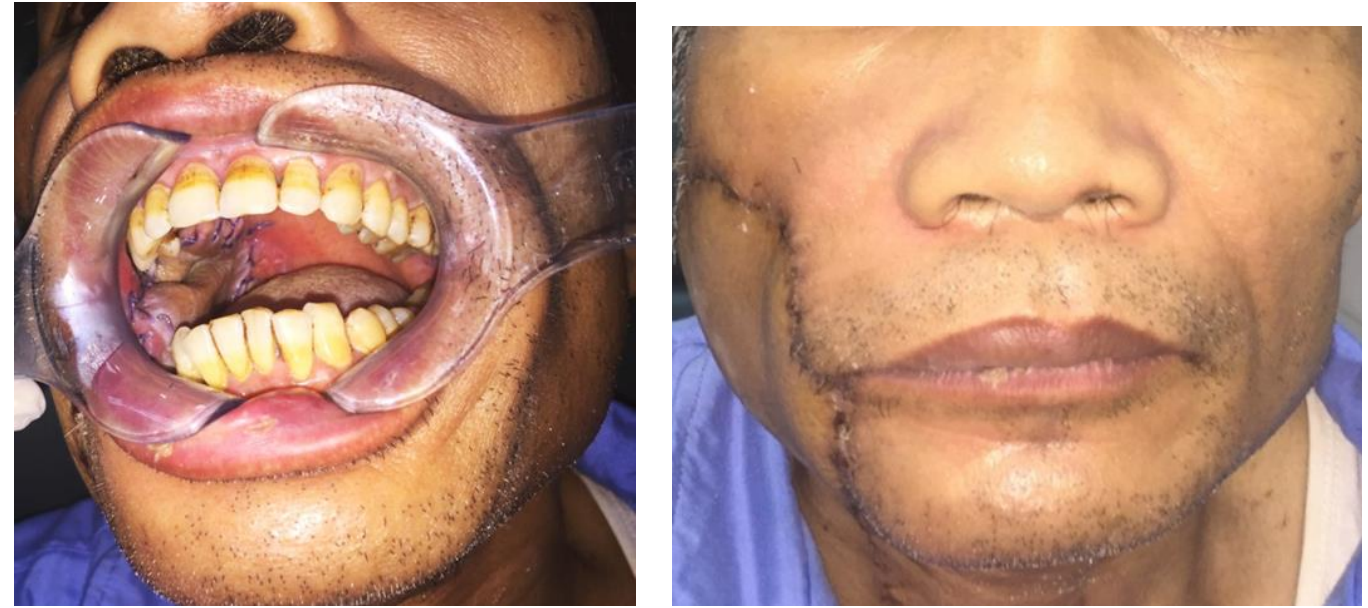

Figure 3. Mandibular and oral floor reconstruction with skin flap and fibular flap. Anterolateral thigh flap to cover affected area
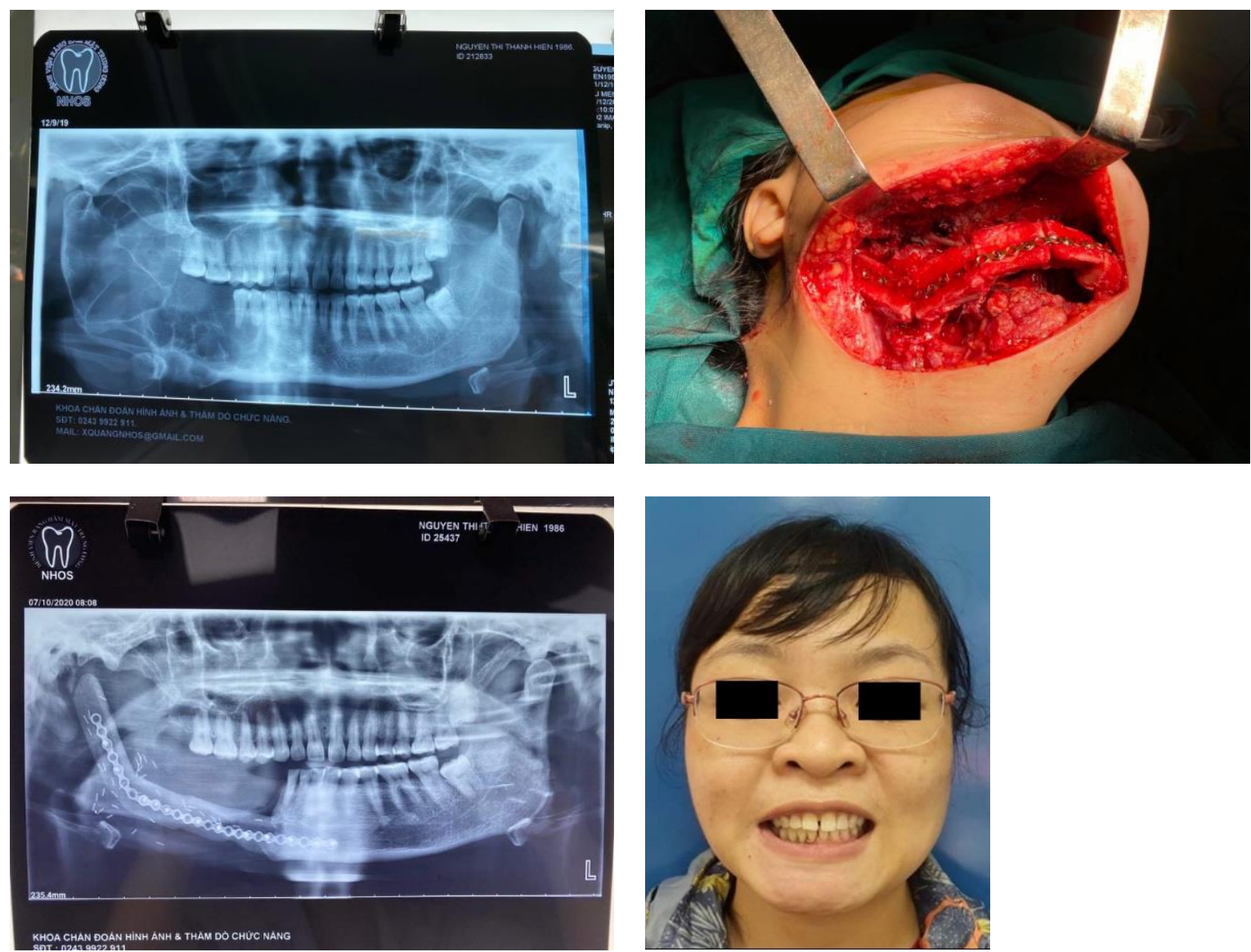

Figure 5. Mandibular reconstruction with fibular flap and 1 year after surgery.

\section{CONCLUSIONS}

Reconstructive surgery following the resection of maxillomandibular lesion is very important. Excision of large benign tumors and malignant tumors always leaves major defects that cannot be repaired with 
conventional reconstructive techniques. Free flap reconstruction is a flexible reconstructive option that can overcome the limitation of conventional approach and restore the essential functions and aesthetics of the facial. The implementation of microsurgery technique at Hanoi National Hospital of Odonto-Stomatology has been carried out for only 12 years but rapid and significant progress has been made.

\section{REFERENCES}

1. Joseph A.R., James J.S., Richard C.K. Oral Pathology: Clinical Pathologic Correlations, 5th, W. B. Saunders, Missouri, USA; 607-705. 2008.

2. Pablo H. Montero, Snehal G. Patel. Cancer of the oral cavity. Surg Oncol Clin N Am. 2015;24(3): 17.

3. R.J. Fonseca. Oral and maxillofacial surgery. 3 ed. R.J. Fonseca, editor2018.

4. Miranda-Filho A, Bray F. Global patterns and trends in cancers of the lip, tongue and mouth. Oral oncology. 2020 Mar;102:104551. PubMed PMID: 31986342.

5. Jatin Shah. Head and Neck surgery and oncology. 5 ed. 5, editor 2020.

6. Andrew M. Fribley. Targeting Oral Cancer. Andrew M. Fribley, editor. Switzerland Springer International; 2016.

7. Rivera C. Essentials of oral cancer. International journal of clinical and experimental pathology. 2015;8(9):11884-94. PubMed PMID: 26617944. Pubmed Central PMCID: 4637760.

8. Neil. S. Norton. Netter's Head and Neck Anatomy for Dentistry. 3 ed2017.

9. William C. Wood, Charley A. Staley, John E. Anatomic basis of tumor surgery. 2, editor: Springer Heidelberg Dordrecht London New York; 2010.

10. J. Craig Merrell, Nguyen Viet Tien, Nguyen Tai Son, Luu Ngoc An, Daniel Sellers, Robert Russell, Ralph Manktelow, Fu-Chan Wei, Dennis P. Orgill. Introduction of Microsurgery in Vietnam by a Charitable Organization: A 15-Year Experience. Plast. Reconstr. Surg. 119: 1267, 2007. 